\title{
Probing the predictions of an orbifold theory of flavor
}

\author{
Francisco J. de Anda๑, ${ }^{1, *}$ Newton Nath $\odot,{ }^{2, \dagger}$ José W. F. Valle, ${ }^{3, \ddagger}$ and Carlos A. Vaquera-Araujo ${ }^{4,5, \S}$ \\ ${ }^{1}$ Tepatitlán's Institute for Theoretical Studies, C.P. 47600, Jalisco, México \\ ${ }^{2}$ Instituto de Física, Universidad Nacional Autónoma de México, A.P. 20-364, \\ Ciudad de México 01000, México \\ ${ }^{3}$ AHEP Group, Institut de Física Corpuscular-C.S.I.C./Universitat de València, \\ Parc Científic de Paterna, C/ Catedrático José Beltrán, 2 E-46980 Paterna, Valencia, Spain \\ ${ }^{4}$ Consejo Nacional de Ciencia y Tecnología, Avenida de los Insurgentes Sur 1582, Colonia Crédito \\ Constructor, Delegación Benito Juárez, C.P. 03940, Ciudad de México, México \\ ${ }^{5}$ Departamento de Física, DCI, Campus León, Universidad de Guanajuato, Loma del Bosque 103, \\ Lomas del Campestre, C.P. 37150, León, Guanajuato, México
}

(Received 17 April 2020; accepted 2 June 2020; published 16 June 2020)

\begin{abstract}
We examine the implications of a recently proposed theory of fermion masses and mixings in which an $A_{4}$ family symmetry emerges from orbifold compactification. We analyze two variant schemes concerning their predictions for neutrino oscillations, neutrinoless double-beta decay, and the golden quark-lepton unification mass relation. We find that upcoming experiments DUNE as well as LEGEND and nEXO offer good chances of exploring a substantial region of neutrino parameters.
\end{abstract}

DOI: 10.1103/PhysRevD.101.116012

\section{INTRODUCTION}

The discovery of neutrino oscillations [1,2] has prompted a great experimental effort toward precision measurements [3]. Indeed, the pattern of neutrino mass and mixing parameters is strikingly at odds with the one that characterizes the quark sector, suggesting that it can hardly be expected to happen just by chance. The most popular approach to bring a rationale to the pattern of neutrino mixing involves the idea that there is some non-Abelian family symmetry in nature. In a modelindependent way, one may assume the existence of some residual $C P$ symmetry characterizing the neutrino mass matrix, irrespective of the details of the underlying theory [4-6]. A more ambitious approach is, of course, to guess what the family symmetry actually is and to build explicit flavor models on a case-by-case basis [7-11]. However, pinning down the nature of such symmetry among the plethora of possibilities is a formidable task.

An interesting theoretical idea has been to imagine the existence of new dimensions in space-time, as a way to

\footnotetext{
*fran@tepaits.mx

†newton@fisica.unam.mx

*alle@ific.uv.es

${ }^{\S}$ vaquera@ fisica.ugto.mx
}

Published by the American Physical Society under the terms of the Creative Commons Attribution 4.0 International license. Further distribution of this work must maintain attribution to the author(s) and the published article's title, journal citation, and DOI. Funded by SCOAP. shed light on the possible nature of the family symmetry in four dimensions. In this context, six-dimensional theories compactified on a torus have been suggested $[12,13]$ and a realistic standard model extension has recently been proposed [14] in which fermions are nicely arranged within the framework of an $A_{4}$ family symmetry. The theory yields very good predictions for fermion masses and mixings, including the "golden" quark-lepton unification formula [15-19].

In this work we focus on the possibility of probing the implications of this theory within the next generation of neutrino experiments. This includes the long-baseline Deep Underground Neutrino Experiment (DUNE) [20,21], as well as neutrinoless double-beta decay ( $0 \nu \beta \beta$ for short) searches. In Sec. II, we describe the theory framework, identifying two model setups, while in Sec. III we determine the potential of upcoming neutrino experiments, such as DUNE and $0 \nu \beta \beta$ experiments to probe our orbifold compactification predictions.

\section{THEORY FRAMEWORK}

Our model features a six-dimensional version of the standard model $S U(3) \otimes S U(2) \otimes U(1)$ gauge symmetry, together with three right-handed neutrinos and supplemented with the orbifold compactification described in our previous paper [14]. The transformation properties of the fields under the gauge and $A_{4}$ family symmetry and their localization on the orbifold are shown in Table I.

The scalar sector consists of three Higgs doublets and an extra singlet scalar $\sigma$, all transforming as flavor triplets. 
TABLE I. Field content of the model.

\begin{tabular}{lcccccc}
\hline \hline Field & $S U(3)$ & $S U(2)$ & $U(1)$ & $A_{4}$ & $\mathbb{Z}_{3}$ & Localization \\
\hline$L$ & $\mathbf{1}$ & $\mathbf{2}$ & $-1 / 2$ & $\mathbf{3}$ & $\omega^{2}$ & Brane \\
$d^{c}$ & $\overline{\mathbf{3}}$ & $\mathbf{1}$ & $1 / 3$ & $\mathbf{3}$ & $\omega$ & Brane \\
$e^{c}$ & $\mathbf{1}$ & $\mathbf{1}$ & 1 & $\mathbf{3}$ & $\omega$ & Brane \\
$Q$ & $\mathbf{3}$ & $\mathbf{2}$ & $1 / 6$ & $\mathbf{3}$ & $\omega^{2}$ & Brane \\
$u_{1,2,3}^{c}$ & $\overline{\mathbf{3}}$ & $\mathbf{1}$ & $-2 / 3$ & $\mathbf{1}^{\prime \prime}, \mathbf{1}^{\prime}, \mathbf{1}$ & $\omega^{2}$ & Bulk \\
$\nu^{c}$ & $\mathbf{1}$ & $\mathbf{1}$ & 0 & $\mathbf{3}$ & 1 & Brane \\
$H_{u}$ & $\mathbf{1}$ & $\mathbf{2}$ & $1 / 2$ & $\mathbf{3}$ & $\omega^{2}$ & Brane \\
$H_{d}$ & $\mathbf{1}$ & $\mathbf{2}$ & $-1 / 2$ & $\mathbf{3}$ & 1 & Brane \\
$H_{\nu}$ & $\mathbf{1}$ & $\mathbf{2}$ & $1 / 2$ & $\mathbf{3}$ & $\omega$ & Brane \\
$\sigma$ & $\mathbf{1}$ & $\mathbf{1}$ & 0 & $\mathbf{3}$ & 1 & Bulk \\
\hline \hline
\end{tabular}

They are charged under a $\mathbb{Z}_{3}$ symmetry, so that $H_{d}$ only couples to down-type fermions (charged leptons and down quarks), $H_{u}$ couples only to up quarks, and $H_{\nu}$ only couples to neutrinos.

The effective Yukawa terms are given by

$$
\begin{aligned}
\mathcal{L}_{Y}= & y^{N} \nu^{c} \nu^{c} \sigma+y_{1}^{\nu}\left(L H_{\nu} \nu^{c}\right)_{1}+y_{2}^{\nu}\left(L H_{\nu} \nu^{c}\right)_{2} \\
& +y_{1}^{d}\left(Q d^{c} H_{d}\right)_{1}+y_{2}^{d}\left(Q d^{c} H_{d}\right)_{2}+y_{1}^{e}\left(L e^{c} H_{d}\right)_{1} \\
& +y_{2}^{e}\left(L e^{c} H_{d}\right)_{2}+y_{1}^{u}\left(Q H_{u}\right)_{1^{\prime}} u_{1}^{c}+y_{2}^{u}\left(Q H_{u}\right)_{1^{\prime \prime}} u_{2}^{c} \\
& +y_{3}^{u}\left(Q H_{u}\right)_{1} u_{3}^{c},
\end{aligned}
$$

where the symbol ()$_{1,2}$ indicates the possible singlet contractions $\mathbf{3} \times \mathbf{3} \times \mathbf{3} \rightarrow \mathbf{1}_{1,2}$ and $\mathbf{3} \times \mathbf{3} \rightarrow \mathbf{1}_{1,1^{\prime}, 1^{\prime \prime}}$ in $A_{4}$. All dimensionless Yukawa couplings are assumed to be real due to a $C P$ symmetry.

The scalar field $\sigma$ gets a vacuum expected value (VEV) that breaks spontaneously lepton number and the $A_{4}$ family symmetry, giving large Majorana masses to the righthanded neutrinos. The corresponding VEV is aligned as

$$
\langle\sigma\rangle=v_{\sigma}\left(\begin{array}{c}
1 \\
\omega \\
\omega^{2}
\end{array}\right)
$$

with $\omega=e^{2 \pi i / 3}$, the cube root of unity.

As $A_{4}$ is broken at a high mass scale, the Higgs doublets can obtain the most general spontaneous $C P$ violating alignment, which we parametrize as

$$
\begin{aligned}
& \left\langle H_{u}\right\rangle=v_{u}\left(\begin{array}{c}
\epsilon_{1}^{u} e^{i \phi_{1}^{u}} \\
\epsilon_{2}^{u} e^{i \phi_{2}^{u}} \\
1
\end{array}\right), \quad\left\langle H_{\nu}\right\rangle=v_{\nu} e^{i \phi^{\nu}}\left(\begin{array}{c}
\epsilon_{1}^{\nu} e^{i \phi_{1}^{\nu}} \\
\epsilon_{2}^{\nu} e^{i \phi_{2}^{\nu}} \\
1
\end{array}\right), \\
& \left\langle H_{d}\right\rangle=v_{d} e^{i \phi^{d}}\left(\begin{array}{c}
\epsilon_{1}^{d} e^{i \phi_{1}^{d}} \\
\epsilon_{2}^{d} e^{i \phi_{2}^{d}} \\
1
\end{array}\right) .
\end{aligned}
$$

An important prediction of the model comes from the fact that the charged leptons and down quarks obtain their masses from the same $H_{d}$, so that the $A_{4}$ structure implies the golden relation between their masses [15]

$$
\frac{m_{\tau}}{\sqrt{m_{\mu} m_{e}}}=\frac{m_{b}}{\sqrt{m_{s} m_{d}}} .
$$

This relation is in good agreement with experiments [22] and is rather robust against renormalization group running.

The explicit form of the mass matrices for the matter fields (up to unphysical rephasings) is given as

$$
\begin{aligned}
& M_{u}=v_{u}\left(\begin{array}{ccc}
y_{1}^{u} \epsilon_{1}^{u} & y_{2}^{u} \epsilon_{1}^{u} & y_{3}^{u} \epsilon_{1}^{u} \\
y_{1}^{u} \epsilon_{2}^{u} \omega^{2} & y_{2}^{u} \epsilon_{2}^{u} \omega & y_{3}^{u} \epsilon_{2}^{u} \\
y_{1}^{u} \omega & y_{2}^{u} \omega^{2} & y_{3}^{u}
\end{array}\right), \\
& M_{d}=v_{d}\left(\begin{array}{ccc}
0 & y_{1}^{d} \epsilon_{1}^{d} e^{i\left(\phi_{1}^{d}-\phi_{2}^{d}\right)} & y_{2}^{d} \epsilon_{2}^{d} \\
y_{2}^{d} \epsilon_{1}^{d} e^{i\left(\phi_{1}^{d}-\phi_{2}^{d}\right)} & 0 & y_{1}^{d} \\
y_{1}^{d} \epsilon_{2}^{d} & y_{2}^{d} & 0
\end{array}\right), \\
& M_{e}=v_{d}\left(\begin{array}{ccc}
y_{2}^{e} \epsilon_{1}^{d} e^{-i\left(\phi_{d}^{1}-\phi_{2}^{d}\right)} & y_{1}^{e} \epsilon_{1}^{d} e^{-i\left(\phi_{1}^{d}-\phi_{2}^{d}\right)} & y_{2}^{e} \epsilon_{2}^{d} \\
y_{1}^{e} \epsilon_{2}^{d} & 0 & y_{1}^{e} \\
M_{N}^{R} & =y^{N} v_{\sigma}\left(\begin{array}{ccc}
\omega^{2} & \omega \\
\omega^{2} & 0 & 1 \\
\omega & 1 & 0
\end{array}\right), & 0 \\
0 & y_{1}^{\nu} \epsilon_{1}^{\nu} e^{i\left(\phi_{1}^{\nu}-\phi_{2}^{\nu}\right)} & y_{2}^{\nu} \epsilon_{2}^{\nu} \\
M_{\nu}^{D} & =v_{\nu}\left(\begin{array}{ccc}
y_{2}^{\nu} \epsilon_{1}^{\nu} e^{i\left(\phi_{1}^{\nu}-\phi_{2}^{\nu}\right)} & 0 & y_{1}^{\nu} \\
y_{1}^{\nu} \epsilon_{2}^{\nu} & y_{2}^{\nu} & 0
\end{array}\right), \\
M_{\nu}^{L} & =M_{\nu}^{D}\left(M_{N}^{R}\right)^{-1}\left(M_{\nu}^{D}\right)^{T} . &
\end{array}\right.
\end{aligned}
$$

In what follows, we adopt the standard parametrization for the Cabibbo-Kobayashi-Maskawa (CKM) matrix,

$$
V_{\mathrm{CKM}}=\left(\begin{array}{ccc}
c_{12}^{q} c_{13}^{q} & s_{12}^{q} c_{13}^{q} & s_{13}^{q} e^{-i \delta^{q}} \\
-s_{12}^{q} c_{23}^{q}-c_{12}^{q} s_{13}^{q} s_{23}^{q} e^{i \delta^{q}} & c_{12}^{q} c_{23}^{q}-s_{12}^{q} s_{13}^{q} s_{23}^{q} e^{i \delta^{q}} & c_{13}^{q} s_{23}^{q} \\
s_{12}^{q} s_{23}^{q}-c_{12}^{q} s_{13}^{q} c_{23}^{q} e^{i \delta^{q}} & -c_{12}^{q} s_{23}^{q}-s_{12}^{q} s_{13}^{q} c_{23}^{q} e^{i \delta^{q}} & c_{13}^{q} c_{23}^{q}
\end{array}\right)
$$


and the symmetrical presentation of the lepton mixing matrix [23,24],

$$
K=\left(\begin{array}{ccc}
c_{12}^{\ell} c_{13}^{\ell} & s_{12}^{\ell} c_{13}^{\ell} e^{-i \phi_{12}} & s_{13}^{\ell} e^{-i \phi_{13}} \\
-s_{12}^{\ell} c_{23}^{\ell} e^{i \phi_{12}}-c_{12}^{\ell} s_{13}^{\ell} s_{23}^{\ell} e^{-i\left(\phi_{23}-\phi_{13}\right)} & c_{12}^{\ell} c_{23}^{\ell}-s_{12}^{\ell} s_{13}^{\ell} s_{23}^{\ell} e^{-i\left(\phi_{23}+\phi_{12}-\phi_{13}\right)} & c_{13}^{\ell} s_{23}^{\ell} e^{-i \phi_{23}} \\
s_{12}^{\ell} s_{23}^{\ell} e^{i\left(\phi_{23}+\phi_{12}\right)}-c_{12}^{\ell} s_{13}^{\ell} c_{23}^{\ell} e^{i \phi_{13}} & -c_{12}^{\ell} s_{23}^{\ell} e^{i \phi_{23}}-s_{12}^{\ell} s_{13}^{\ell} c_{23}^{\ell} e^{-i\left(\phi_{12}-\phi_{13}\right)} & c_{13}^{\ell} c_{23}^{\ell}
\end{array}\right),
$$

with $c_{i j}^{f} \equiv \cos \theta_{i j}^{f}$ and $s_{i j}^{f} \equiv \sin \theta_{i j}^{f}$, where $f=q, \ell$. The advantage of using the symmetrical parametrization for the lepton mixing matrix resides in the transparent role of the Majorana phases in the effective mass parameter characterizing the amplitude for neutrinoless double-beta decay

$$
\left\langle m_{\beta \beta}\right\rangle=\left|\sum_{j=1}^{3} K_{e j}^{2} m_{j}\right|=\left|c_{12}^{\ell 2} c_{13}^{\ell 2} m_{1}+s_{12}^{\ell 2} c_{13}^{\ell 2} m_{2} e^{2 i \phi_{12}}+s_{13}^{\ell 2} m_{3} e^{2 i \phi_{13}}\right|,
$$

while keeping a rephasing-invariant expression for the Dirac phase

$$
\delta^{\ell}=\phi_{13}-\phi_{12}-\phi_{23}
$$

which affects neutrino oscillation probabilities.

\section{A. Model Setup I (MI)}

Following [14], we may further assume that the Higgs VEVs preserve conventional (trivial) $C P$ symmetry, and therefore, they are real. Together with the reality of the Yukawa couplings, this implies that the only source of $C P$ violation is the phase $\omega$. This leads to a very strong predictivity.

The model is specified by 15 parameters $\left(y_{1,2}^{\nu} v_{\nu}, y_{1,2}^{e, d} v_{d}\right.$, $\left.y_{1,2,3}^{u} v_{u}, \epsilon_{1,2}^{u, \nu, d}\right)$ that describe 22 low-energy flavor observables: $\left(m_{u, c, t, d, s, b, e, \mu, \tau}, m_{1,2,3}^{\nu}, \theta_{12,13,23}^{q}, \delta^{q}, \theta_{12,13,23}^{l}, \phi_{12,13,23}\right)$,

TABLE II. Best-fit values of the model parameters of MI. Here $C P$ violation is generated by a fixed phase $\omega$.

\begin{tabular}{lr}
\hline \hline Parameter & Value \\
\hline$y_{1}^{e} v_{d} / \mathrm{GeV}$ & 1.745 \\
$y_{2}^{e} v_{d} /\left(10^{-1} \mathrm{GeV}\right)$ & -1.019 \\
$y_{1}^{d} v_{d} /\left(10^{-2} \mathrm{GeV}\right)$ & -4.690 \\
$y_{2}^{d} v_{d} / \mathrm{GeV}$ & 2.88 \\
$y_{1}^{\nu} v_{\nu} / \sqrt{Y^{N} v_{\sigma} \mathrm{meV} \times 10^{-1}}$ & 7.54 \\
$y_{2}^{\nu} v_{\nu} /\left(\sqrt{Y^{N} v_{\sigma} \mathrm{meV}} \times 10^{-3}\right)$ & 1.89 \\
$y_{1}^{u} v_{u} /\left(10^{-1} \mathrm{GeV}\right)$ & 6.24 \\
$y_{2}^{u} v_{u} /\left(10^{2} \mathrm{GeV}\right)$ & 1.71 \\
$y_{3}^{u} v_{u} / \mathrm{GeV}$ & -7.13 \\
$\epsilon_{1}^{u} / 10^{-4}$ & -6.90 \\
$\epsilon_{2}^{u} / 10^{-2}$ & 6.24 \\
$\epsilon_{1}^{d} / 10^{-3}$ & -2.74 \\
$\epsilon_{2}^{d} / 10^{-3}$ & 6.00 \\
$\epsilon_{1}^{\nu}$ & 1.16 \\
$\epsilon_{2}^{\nu} / 10^{-1}$ & -3.23 \\
\hline \hline
\end{tabular}

including the neutrino Majorana phases. One extra parameter $\left(y^{N} v_{\sigma}\right)$ defines the masses of the three right-handed neutrinos.

One can perform a global fit to the flavor observables by defining the chi-square function

$$
\chi^{2}=\sum\left(\mu_{\exp }-\mu_{\text {model }}\right)^{2} / \sigma_{\exp }^{2}
$$

\begin{tabular}{|c|c|c|c|}
\hline \multirow[b]{2}{*}{ Observable } & \multicolumn{2}{|c|}{ Data } & \multirow[b]{2}{*}{ Model best fit } \\
\hline & Central value & $1 \sigma$ range & \\
\hline$\theta_{12}^{\ell} /{ }^{\circ}$ & 34.44 & $33.46 \rightarrow 35.67$ & 34.36 \\
\hline$\theta_{13}^{\ell} /^{\circ}$ & 8.45 & $8.31 \rightarrow 8.61$ & 8.31 \\
\hline$\theta_{23}^{\ell} /{ }^{\circ}$ & 47.69 & $45.97 \rightarrow 48.85$ & 48.47 \\
\hline$\delta^{\ell} /^{\circ}$ & 237 & $210 \rightarrow 275$ & 268 \\
\hline$m_{e} / \mathrm{MeV}$ & 0.489 & $0.489 \rightarrow 0.489$ & 0.489 \\
\hline$m_{\mu} / \mathrm{GeV}$ & 0.102 & $0.102 \rightarrow 0.102$ & 0.102 \\
\hline$m_{\tau} / \mathrm{GeV}$ & 1.745 & $1.743 \rightarrow 1.747$ & 1.745 \\
\hline$\Delta m_{21}^{2} /\left(10^{-5} \mathrm{eV}^{2}\right)$ & 7.55 & $7.39 \rightarrow 7.75$ & 7.63 \\
\hline$\Delta m_{31}^{2} /\left(10^{-3} \mathrm{eV}^{2}\right)$ & 2.50 & $2.47 \rightarrow 2.53$ & 2.42 \\
\hline$m_{1} / \mathrm{meV}$ & & & 4.12 \\
\hline$m_{2} / \mathrm{meV}$ & & & 9.66 \\
\hline$m_{3} / \mathrm{meV}$ & & & 50.11 \\
\hline$\phi_{12} /^{\circ}$ & & & 250 \\
\hline$\phi_{13} /^{\circ}$ & & & 187 \\
\hline$\phi_{23} /{ }^{\circ}$ & & & 29 \\
\hline$\theta_{12}^{q} /^{\circ}$ & 13.04 & $12.99 \rightarrow 13.09$ & 13.04 \\
\hline$\theta_{13}^{q} /^{\circ}$ & 0.20 & $0.19 \rightarrow 0.22$ & 0.20 \\
\hline$\theta_{23}^{q} /{ }^{\circ}$ & 2.38 & $2.32 \rightarrow 2.44$ & 2.37 \\
\hline$\delta^{q} /^{\circ}$ & 68.75 & $64.25 \rightarrow 73.25$ & 60.25 \\
\hline$m_{u} / \mathrm{MeV}$ & 1.28 & $0.76 \rightarrow 1.55$ & 1.29 \\
\hline$m_{c} / \mathrm{GeV}$ & 0.626 & $0.607 \rightarrow 0.645$ & 0.626 \\
\hline$m_{t} / \mathrm{GeV}$ & 171.6 & $170 \rightarrow 173$ & 171.6 \\
\hline$m_{d} / \mathrm{MeV}$ & 2.74 & $2.57 \rightarrow 3.15$ & 2.75 \\
\hline$m_{s} / \mathrm{MeV}$ & 54 & $51 \rightarrow 57$ & 51 \\
\hline$m_{b} / \mathrm{GeV}$ & 2.85 & $2.83 \rightarrow 2.88$ & 2.91 \\
\hline$\chi^{2}$ & & & 12.4 \\
\hline
\end{tabular}

TABLE III. Global best-fit of flavor observables within MI. 
where the sum runs through the 19 measured physical parameters (note that the overall neutrino mass scale and the two Majorana phases are currently undetermined). We make use of the MPT package [25] to obtain the flavor observables from the mass matrices in Eq. (5). Then we scan the 15 free parameters and find the values that minimize the $\chi^{2}$ function. Neutrino oscillation parameters are taken from the global fit in Ref. [3], while the rest of the observables are taken from the Particle Data Group [22]. For consistency of the fit, all quark and charged lepton masses are evolved to the same common scale, which we choose to be $M_{Z}$. The running of CKM and neutrino mixing parameters is negligible [26,27]. The results are shown in Tables II and III. One sees from the fit that $\chi^{2}=12.4$. This indicates a relatively good global fit, with some tension in the description of quark $C P$ violation, as seen from the tables. The origin for this is traced to the absence of a free parameter describing $C P$ violation, as discussed above.

\section{B. Model Setup II (MII)}

We can now relax the assumption that all the Higgs VEVs are real, allowing them to be general complex numbers. However, we keep the assumption that the Yukawa couplings are real. This reinstates two physical phases $\phi_{1}^{\nu, d}-\phi_{2}^{\nu, d}$, now increasing the number of free parameters to 17 , for a total of 22 flavor observables.

In this general setup, we loose predictivity for the physical $C P$ violating phases $\delta^{l, q}$, leading to a drastic improvement of the global fit, achieving a minimum $\chi^{2}=$ 1.6 as seen in Tables IV and V. Notice now the very good

TABLE IV. Best-fit values of the model parameters of MII. Here there are two free $C P$ violation phases.

\begin{tabular}{lr}
\hline \hline Parameter & Value \\
\hline$y_{1}^{e} v_{d} /\left(10^{-1} \mathrm{GeV}\right)$ & -1.020 \\
$y_{2}^{e} v_{d} / \mathrm{GeV}$ & 1.745 \\
$y_{1}^{d} v_{d} /\left(10^{-2} \mathrm{GeV}\right)$ & -5.069 \\
$y_{2}^{d} v_{d} / \mathrm{GeV}$ & 2.869 \\
$y_{1}^{\nu} v_{\nu} / \sqrt{Y^{N} v_{\sigma} \mathrm{meV}}$ & -1.461 \\
$y_{2}^{\nu} v_{\nu} / \sqrt{Y^{N} v_{\sigma} \mathrm{meV}}$ & 7.647 \\
$y_{1}^{u} v_{u} /\left(10^{-1} \mathrm{GeV}\right)$ & 6.198 \\
$y_{2}^{u} v_{u} /\left(10^{2} \mathrm{GeV}\right)$ & 1.712 \\
$y_{3}^{u} v_{u} / \mathrm{GeV}$ & 7.143 \\
$\epsilon_{1}^{u} / 10^{-4}$ & -6.926 \\
$\epsilon_{2}^{u} / 10^{-2}$ & -5.058 \\
$\epsilon_{1}^{d} / 10^{-3}$ & 2.812 \\
$\epsilon_{2}^{d} / 10^{-3}$ & 5.863 \\
$\epsilon_{1}^{\nu} / 10^{-1}$ & -9.950 \\
$\epsilon_{2}^{\nu} / 10^{-1}$ & 5.979 \\
$\left(\phi_{1}^{d}-\phi_{2}^{d}\right) / \pi$ & -1.078 \\
$\left(\phi_{1}^{\nu}-\phi_{2}^{\nu}\right) / \pi$ & 1.093 \\
\hline \hline
\end{tabular}

agreement of all of the observables, including the value of the CKM $C P$ violation phase $\delta^{q}$.

As mentioned above, a characteristic feature of our schemes is the golden quark-lepton mass relation given in Eq. (4). We now turn to study this prediction as obtained from our global fits of flavor observables within models I and II. In Fig. 1 we use the golden quark-lepton mass relation in MI and MII to make predictions for the downand strange-quark masses. Here the cyan bands stand for the 1,2 , and $3 \sigma$ regions compatible with the exact golden relation $m_{\tau} / \sqrt{m_{\mu} m_{e}}=m_{b} / \sqrt{m_{s} m_{d}}$ at the $M_{Z}$ scale, and the yellow contours are the 1,2 , and $3 \sigma$ regions for the quark mass parameters measured at the same scale. To better appreciate the predictive power of our framework, we have varied randomly the parameters of MII around the best-fit point in Tables IV and V, and we have determined the shape of the parameter region consistent at $3 \sigma$ with all the 19 measured parameters of the model. This region is shown in purple in Fig. 1. The corresponding contour for MI is not shown as it is very similar, given the fact that the golden relation is not very sensitive to the improvement of the $C P$ violating phases in MII, compared to MI. However, one can see that the best-fit point for scheme MII, indicated

TABLE V. Global best-fit of flavor observables within MII.

\begin{tabular}{|c|c|c|c|}
\hline \multirow[b]{2}{*}{ Observable } & \multicolumn{2}{|c|}{ Data } & \multirow[b]{2}{*}{ Model best fit } \\
\hline & Central value & $1 \sigma$ range & \\
\hline$\theta_{12}^{\ell} /{ }^{\circ}$ & 34.44 & $33.4 \rightarrow 35.67$ & 34.65 \\
\hline$\theta_{13}^{\ell} /{ }^{\circ}$ & 8.45 & $8.31 \rightarrow 8.61$ & 8.44 \\
\hline$\theta_{23}^{\ell} /{ }^{\circ}$ & 47.69 & $45.97 \rightarrow 48.85$ & 47.56 \\
\hline$\delta^{\ell} /^{\circ}$ & 237 & $210 \rightarrow 275$ & 198.3 \\
\hline$m_{e} / \mathrm{MeV}$ & 0.489 & $0.489 \rightarrow 0.489$ & 0.489 \\
\hline$m_{\mu} / \mathrm{GeV}$ & 0.102 & $0.102 \rightarrow 0.102$ & 0.102 \\
\hline$m_{\tau} / \mathrm{GeV}$ & 1.745 & $1.743 \rightarrow 1.747$ & 1.745 \\
\hline$\Delta m_{21}^{2} /\left(10^{-5} \mathrm{eV}^{2}\right)$ & 7.55 & $7.39 \rightarrow 7.75$ & 7.55 \\
\hline$\Delta m_{31}^{2} /\left(10^{-3} \mathrm{eV}^{2}\right)$ & 2.50 & $2.47 \rightarrow 2.53$ & 2.42 \\
\hline$m_{1} / \mathrm{meV}$ & & & 24.31 \\
\hline$m_{2} / \mathrm{meV}$ & & & 25.81 \\
\hline$m_{3} / \mathrm{meV}$ & & & 55.60 \\
\hline$\phi_{12} /^{\circ}$ & & & 252.5 \\
\hline$\phi_{13} /^{\circ}$ & & & 142.3 \\
\hline$\phi_{23} /^{\circ}$ & & & 51.5 \\
\hline$\theta_{12}^{q} /{ }^{\circ}$ & 13.04 & $12.99 \rightarrow 13.09$ & 13.04 \\
\hline$\theta_{13}^{q} /{ }^{\circ}$ & 0.20 & $0.19 \rightarrow 0.22$ & 0.20 \\
\hline$\theta_{23}^{q} /{ }^{\circ}$ & 2.38 & $2.32 \rightarrow 2.44$ & 2.38 \\
\hline$\delta^{q} /^{\circ}$ & 68.75 & $64.25 \rightarrow 73.25$ & 69.25 \\
\hline$m_{u} / \mathrm{MeV}$ & 1.28 & $0.76 \rightarrow 1.81$ & 1.29 \\
\hline$m_{c} / \mathrm{GeV}$ & 0.626 & $0.607 \rightarrow 0.645$ & 0.626 \\
\hline$m_{t} / \mathrm{GeV}$ & 171.6 & $170 \rightarrow 173$ & 171.6 \\
\hline$m_{d} / \mathrm{MeV}$ & 2.74 & $2.35 \rightarrow 3.15$ & 2.51 \\
\hline$m_{s} / \mathrm{MeV}$ & 54 & $51 \rightarrow 57$ & 54 \\
\hline$m_{b} / \mathrm{GeV}$ & 2.85 & $2.76 \rightarrow 2.94$ & 2.87 \\
\hline$\chi^{2}$ & & & 1.6 \\
\hline
\end{tabular}




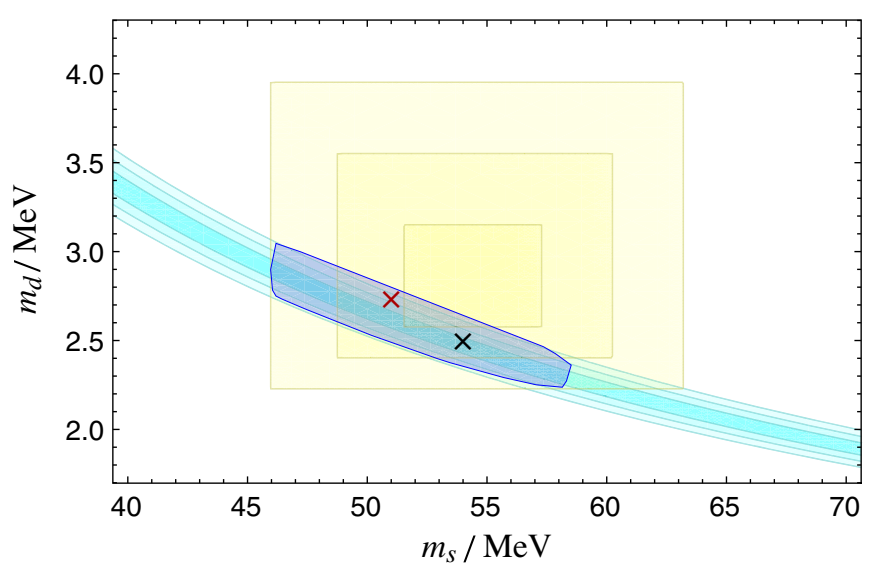

FIG. 1. Prediction for the down- and strange-quark masses at the $M_{Z}$ scale. The cyan contours represent the 1,2 , and $3 \sigma$ allowed regions from the golden relation $m_{\tau} / \sqrt{m_{\mu} m_{e}}=m_{b} /$ $\sqrt{m_{s} m_{d}}$. The yellow contours show the 1,2 , and $3 \sigma$ ranges of the measured quark masses at the $M_{Z}$ scale [27]. The blue region is the allowed parameter space consistent at $3 \sigma$ with the global flavor fit in Tables IV and V. The red (black) cross indicates the location of the best-fit point for MI (MII).

by the black cross, is now compatible at $1 \sigma$ with the exact golden formula.

\section{PROBING NEUTRINO PREDICTIONS}

In this section, we present a close-up of the neutrino predictions of our orbifold compactification schemes, examining also the capability of future experiments to test them.

\section{A. Neutrino oscillations at DUNE}

We start by quantifying the capability of the DUNE experiment to test the oscillation predictions resulting from the $A_{4}$ family symmetry, as realized from a six-dimensional space-time after orbifold compactification. Before presenting details about the simulated results, we first give a brief technical overview of the simulation details of DUNE, the proposed next generation superbeam neutrino oscillation experiment at Fermilab, USA [20,21]. The collaboration plans to use neutrinos from the main injector at Fermilab as a neutrino source. In this experiment, the first detector will record particle interactions near the beam source, at Fermilab. On the other hand, the neutrinos from Fermilab will travel a distance of $1300 \mathrm{~km}$ before reaching the far detector situated at the underground laboratory of the Sanford Underground Research Facility in Lead, South Dakota. The proposed far detector will use four $10 \mathrm{kton}$ volume of liquid argon time-projection chambers. The expected neutrino flux corresponding to $1.07 \mathrm{MW}$ beam power gives $1.47 \times 10^{21}$ protons on target per year for an $80 \mathrm{GeV}$ proton beam energy. We follow the same procedure as given in [28] for performing our numerical analysis of

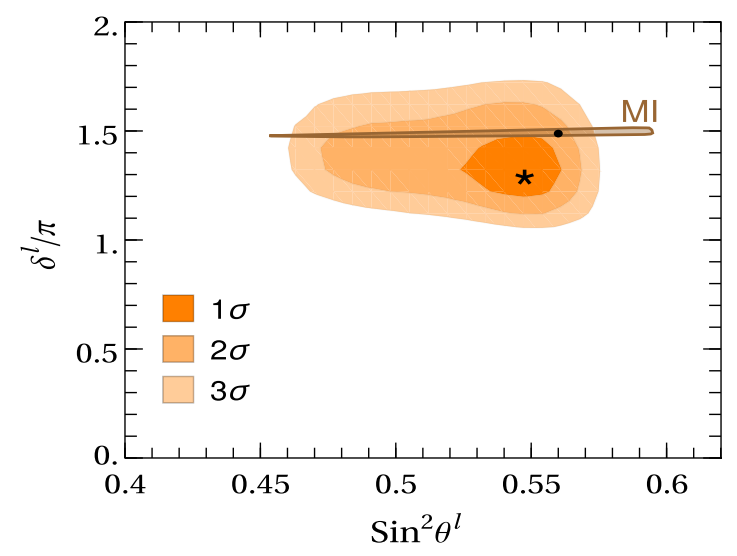

FIG. 2. DUNE sensitivity region in the $\left(\sin ^{2} \theta_{23}^{l}, \delta^{l}\right)$ plane. The asterisk represents the latest neutrino oscillation best fit [3], while the black dot is the predicted best fit, as given in Tables II and III.

DUNE. The GLoBES package [29,30], along with the auxiliary files as mentioned in [21], has been utilized for the simulation. We adopt $3.5 \mathrm{yr}$ running time in both neutrino and antineutrino modes, with a 40 kton total detector volume. In the numerical analysis, we also take into account both the appearance and disappearance channels of neutrinos and antineutrinos. In addition, both the signal and background normalization uncertainties for the appearance as well as disappearance channels have been taken into account in our analysis, as mentioned in the DUNE conceptual design report [21].

Given that normal mass ordering (i.e., $m_{1}<m_{2}<m_{3}$ ) of neutrinos is currently preferred over the inverted one (i.e., $m_{3}<m_{1}<m_{2}$ ) at more than $3 \sigma$ [3], we focus on the first scenario throughout this work.

In what follows, we examine the sensitivity regions of DUNE in the $\left(\sin ^{2} \theta_{23}^{l}, \delta^{l}\right)$ for different seed points. These are shown at $1 \sigma$ (dark orange), $2 \sigma$ (orange), and $3 \sigma$ (lighter orange) confidence level, respectively.

In Fig. 2, we show the expected 1, 2, and $3 \sigma$ DUNE sensitivity regions in the $\left(\sin ^{2} \theta_{23}^{l}, \delta^{l}\right)$ plane. Here we assume model setup MI and take the latest neutrino oscillation best-fit point from [3] as benchmark, as indicated by the black asterisk. The corresponding MI theory predictions are indicated by the brown $3 \sigma$ confidence level region, and its best-fit point, as given in Tables II and III, is shown by the black dot. One sees that DUNE will be able to rule out predicted correlation between $\sin ^{2} \theta_{23}^{l}$ and $\delta^{l}$ for MI at $1 \sigma$ C.L. In contrast, we note that the predicted region in MII covers the full DUNE sensitivity contours, so we do not show this plot in Fig. 2. In other words, if the current best-fit value of the oscillation parameters remains, DUNE will not be able to rule out the predictions for MII even at $1 \sigma$ C.L.

We now change our seed points, adopting as benchmarks the $\left(\sin ^{2} \theta_{23}^{l}, \delta^{l}\right)$ best-fit points predicted in each of the models described above. The resulting DUNE sensitivity regions are given in Fig. 3. 

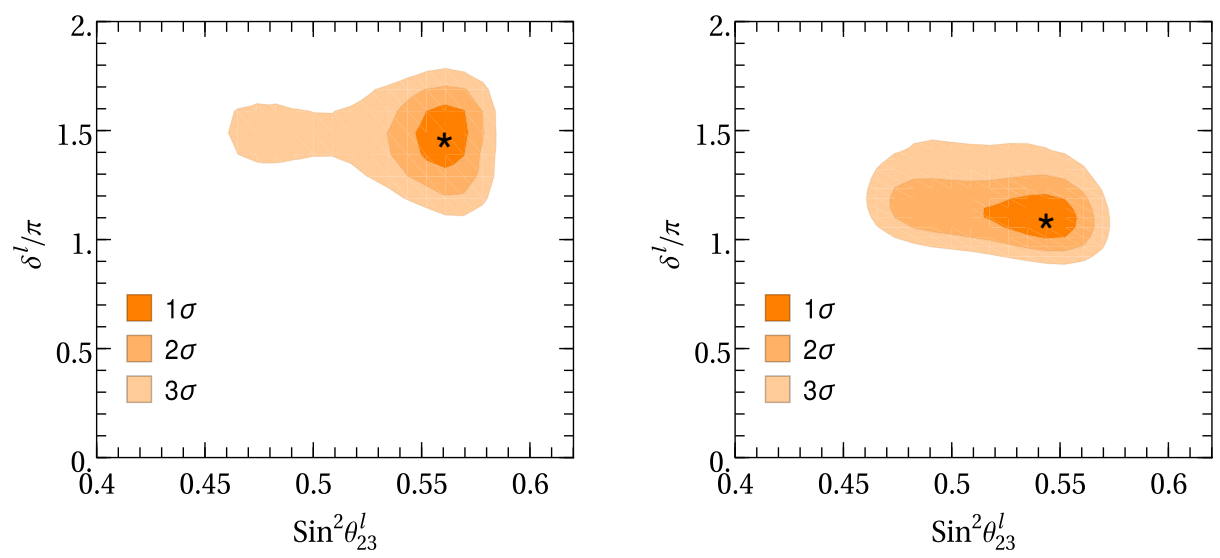

FIG. 3. DUNE $\left(\sin ^{2} \theta_{23}, \delta^{l}\right)$ sensitivity regions in models (left) MI and (right) MII, assuming the corresponding best-fit points obtained in setup (left) MI and (right) MII, respectively, as indicated by the asterisks.

One sees from the left panel that, if the MI predicted value of $\left(\sin ^{2} \theta_{23}^{l}, \delta^{l}\right)$ is the true benchmark value, then DUNE (after 3.5 running time in both neutrino and antineutrino modes) can rule out maximal value of $\theta_{23}^{l}$, i.e., $\sin ^{2} \theta_{23}^{l}=\pi / 2$ at $2 \sigma$ confidence level. On the other hand, by adopting the MII predicted best fit as the true seed value, we notice from the right panel that DUNE can rule out maximal value of $\sin ^{2} \theta_{23}^{l}$ at $1 \sigma$, whereas it can rule out $\delta^{l}=3 \pi / 2$ at $3 \sigma$ confidence level.

\section{B. Neutrinoless double-beta decay}

We now turn to the predictions for neutrinoless doublebeta decay in models MI and MII and confront them with

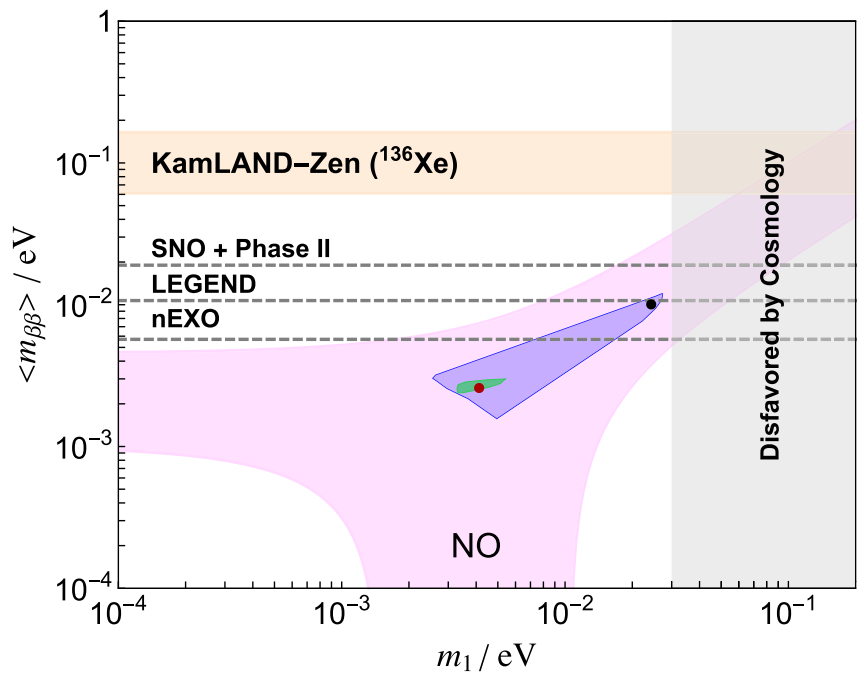

FIG. 4. Effective Majorana neutrino mass parameter $\left\langle m_{\beta \beta}\right\rangle$ as a function of the lightest active neutrino mass $m_{1}$. Here the green (blue) contour represents the predicted $\left\langle m_{\beta \beta}\right\rangle$ parameter space consistent at $3 \sigma$ with the global flavor fit for model setup MI (MII), and the best-fit value is shown by the red (black) dot. The current KamLAND-Zen limit is shown by the light yellow band, and the projected sensitivities for future experiments are indicated in dashed horizontal lines; see text for details. experimental sensitivities [31-38]. This is shown in Fig. 4. The values for the effective mass $\left\langle m_{\beta \beta}\right\rangle$ consistent at $3 \sigma$ with the measured flavor observables (mainly neutrino oscillation parameters) obtained from the global fit are represented by the green contour for the case of the "constrained" model MI and by the blue one for MII. The theory predicted regions are obtained by allowing the free parameters to vary randomly from the best-fit point while simultaneously complying at $3 \sigma$ with all the measured observables of the global fit. One sees that the predicted region for MII becomes wider, while the region for MI remains quite small. This is due to the effect of the variation of the available free phases in MII $\phi_{1}^{\nu, d}-\phi_{2}^{\nu, d}$, which are directly related to the Majorana phases. In contrast, in MI the only available $C P$ violating phase is fixed, leading to sharply predicted $0 \nu \beta \beta$ decay amplitude, which cannot deviate much from its best-fit value.

Interestingly enough, predictivity is not destroyed by the inclusion of those extra phases, and MII still has upper and lower bounds for both the effective mass $\left\langle m_{\beta \beta}\right\rangle$ and the lightest neutrino mass parameter. As a visual guide for the experimental searches of $0 \nu \beta \beta$, in Fig. 4 the horizontal yellow band indicates the current experimental limits from KamLAND-Zen (61-165 meV) [31], while the dashed lines correspond to the most optimistic sensitivities projected for $\mathrm{SNO}+$ Phase II (19-46 meV) [35], LEGEND (10.7-22.8 meV) [36], and nEXO (5.7-17.7 meV) [37]. One sees that the best-fit point for MII, marked with a black point, becomes testable by the next generation of $0 \nu \beta \beta$ experiments LEGEND and nEXO. Finally, the vertical gray band represents the current sensitivity of cosmological data from the Planck Collaboration [39].

\section{CONCLUSION}

We have investigated the implications of a recently proposed theory of fermion masses and mixings based on an $A_{4}$ family symmetry that arises from the compactification of a six-dimensional orbifold. We have analyzed two variations of the idea, a constrained one, in which $C P$ 
violation is strictly predicted, and another where $C P$ phases are free to vary. We have quantified the predictions of these schemes for neutrino oscillations, neutrinoless double-beta decay, and the golden quark-lepton mass formula. We have found that the projected long-baseline experiment DUNE can probe the model predictions concerning the maximality of the atmospheric mixing or the value of the $C P$ phase in a meaningful way. Likewise, the next generation of neutrinoless double-beta decay experiments, especially LEGEND and $\mathrm{nEXO}$, could probe our model MII in a substantial region of parameters.

\section{ACKNOWLEDGMENTS}

Work is supported by the Spanish Grants No. SEV2014-0398 and No. FPA2017-85216-P (AEI/FEDER, UE), PROMETEO/2018/165 (Generalitat Valenciana), and the Spanish Red Consolider MultiDark FPA2017-90566REDC. C. A. V.-A. is supported by the Mexican Cátedras CONACYT Project No. 749 and No. SNI 58928. N. N. is supported by the DGAPA-UNAM postdoctoral fellowship program, CONACYT CB-2017-2018/A1-S-13051 (México) and DGAPA-PAPIIT IN107118.
[1] T. Kajita, Nobel lecture: Discovery of atmospheric neutrino oscillations, Rev. Mod. Phys. 88, 030501 (2016).

[2] A. B. McDonald, Nobel lecture: The Sudbury Neutrino Observatory: Observation of flavor change for solar neutrinos, Rev. Mod. Phys. 88, 030502 (2016).

[3] P. de Salas, D. V. Forero, C. A. Ternes, M. Tórtola, and J. W. F. Valle, Status of neutrino oscillations 2018: $3 \sigma$ hint for normal mass ordering and improved CP sensitivity, Phys. Lett. B 782, 633 (2018).

[4] P. Chen, G.-J. Ding, F. Gonzalez-Canales, and J. W. F. Valle, Generalized $\mu-\tau$ reflection symmetry and leptonic CP violation, Phys. Lett. B 753, 644 (2016).

[5] P. Chen, G.-J. Ding, F. Gonzalez-Canales, and J. W. F. Valle, Classifying $C P$ transformations according to their texture zeros: Theory and implications, Phys. Rev. D 94, 033002 (2016).

[6] P. Chen, S. C. Chuliá, G.-J. Ding, R. Srivastava, and J. W. F. Valle, $C P$ symmetries as guiding posts: Revamping tribimaximal mixing. II, Phys. Rev. D 100, 053001 (2019).

[7] K. Babu, E. Ma, and J. W. F. Valle, Underlying A(4) symmetry for the neutrino mass matrix and the quark mixing matrix, Phys. Lett. B 552, 207 (2003).

[8] G. Altarelli and F. Feruglio, Discrete flavor symmetries and models of neutrino mixing, Rev. Mod. Phys. 82, 2701 (2010).

[9] S. Morisi and J. W. F. Valle, Neutrino masses and mixing: A flavour symmetry roadmap, Fortschr. Phys. 61, 466 (2013).

[10] S. F. King, A. Merle, S. Morisi, Y. Shimizu, and M. Tanimoto, Neutrino mass and mixing: From theory to experiment, New J. Phys. 16, 045018 (2014).

[11] P. Chen, G.-J. Ding, A. D. Rojas, C. A. Vaquera-Araujo, and J.W.F. Valle, Warped flavor symmetry predictions for neutrino physics, J. High Energy Phys. 01 (2016) 007.

[12] F. J. de Anda and S. F. King, An $S_{4} \times S U(5)$ SUSY GUT of flavour in 6d, J. High Energy Phys. 07 (2018) 057.

[13] F. J. de Anda and S. F. King, $S U(3) \times S O(10)$ in 6d, J. High Energy Phys. 10 (2018) 128.

[14] F. J. de Anda, J. W. F. Valle, and C. A. Vaquera-Araujo, Flavour and CP predictions from orbifold compactification, Phys. Lett. B 801, 135195 (2020).
[15] S. Morisi, E. Peinado, Y. Shimizu, and J. W. F. Valle, Relating quarks and leptons without grand unification, Phys. Rev. D 84, 036003 (2011).

[16] S. King, S. Morisi, E. Peinado, and J. W. F. Valle, Quarklepton mass relation in a realistic $A_{4}$ extension of the Standard Model, Phys. Lett. B 724, 68 (2013).

[17] S. Morisi, M. Nebot, K. M. Patel, E. Peinado, and J. W. F. Valle, Quark-lepton mass relation and CKM mixing in an A4 extension of the minimal supersymmetric Standard Model, Phys. Rev. D 88, 036001 (2013).

[18] C. Bonilla, S. Morisi, E. Peinado, and J. W. F. Valle, Relating quarks and leptons with the $T_{7}$ flavour group, Phys. Lett. B 742, 99 (2015).

[19] M. Reig, J. W. Valle, and F. Wilczek, SO(3) family symmetry and axions, Phys. Rev. D 98, 095008 (2018).

[20] R. Acciarri et al. (DUNE Collaboration), Long-Baseline Neutrino Facility (LBNF) and deep underground neutrino experiment (DUNE), arXiv:1512.06148.

[21] T. Alion et al. (DUNE Collaboration), Experiment simulation configurations used in DUNE CDR, arXiv: 1606.09550.

[22] M. Tanabashi et al. (Particle Data Group), Review of particle physics, Phys. Rev. D 98, 030001 (2018).

[23] J. Schechter and J. W. F. Valle, Neutrino masses in $\mathrm{SU}(2) \times$ U(1) theories, Phys. Rev. D 22, 2227 (1980).

[24] W. Rodejohann and J. W. F. Valle, Symmetrical parametrizations of the lepton mixing matrix, Phys. Rev. D 84, 073011 (2011).

[25] S. Antusch, J. Kersten, M. Lindner, M. Ratz, and M. A. Schmidt, Running neutrino mass parameters in see-saw scenarios, J. High Energy Phys. 03 (2005) 024.

[26] Z.-z. Xing, H. Zhang, and S. Zhou, Updated values of running quark and Lepton masses, Phys. Rev. D 77, 113016 (2008).

[27] S. Antusch and V. Maurer, Running quark and lepton parameters at various scales, J. High Energy Phys. 11 (2013) 115.

[28] N. Nath, R. Srivastava, and J. W. F. Valle, Testing generalized $C P$ symmetries with precision studies at DUNE, Phys. Rev. D 99, 075005 (2019).

[29] P. Huber, M. Lindner, and W. Winter, Simulation of longbaseline neutrino oscillation experiments with GLoBES 
(General Long Baseline Experiment Simulator), Comput. Phys. Commun. 167, 195 (2005).

[30] P. Huber, J. Kopp, M. Lindner, M. Rolinec, and W. Winter, New features in the simulation of neutrino oscillation experiments with GLoBES 3.0: General long baseline experiment simulator, Comput. Phys. Commun. 177, 432 (2007).

[31] A. Gando et al. (KamLAND-Zen Collaboration), Search for Majorana Neutrinos near the Inverted Mass Hierarchy Region with KamLAND-Zen, Phys. Rev. Lett. 117, 082503 (2016).

[32] C. Alduino et al. (CUORE Collaboration), First Results from CUORE: A Search for Lepton Number Violation via $0 \nu \beta \beta$ Decay of ${ }^{130} \mathrm{Te}$, Phys. Rev. Lett. 120, 132501 (2018).

[33] J. Albert et al. (EXO Collaboration), Search for Neutrinoless Double-Beta Decay with the Upgraded EXO-200 Detector, Phys. Rev. Lett. 120, 072701 (2018).
[34] M. Agostini et al. (GERDA Collaboration), Improved Limit on Neutrinoless Double- $\beta$ Decay of ${ }^{76} \mathrm{Ge}$ from GERDA Phase II, Phys. Rev. Lett. 120, 132503 (2018).

[35] S. Andringa et al. (SNO+Collaboration), Current status and future prospects of the $\mathrm{SNO}+$ experiment, Adv. High Energy Phys. 2016, 6194250 (2016).

[36] N. Abgrall et al. (LEGEND Collaboration), The large enriched germanium experiment for neutrinoless double beta decay (LEGEND), AIP Conf. Proc. 1894, 020027 (2017).

[37] J. Albert et al. (nEXO Collaboration), Sensitivity and discovery potential of nEXO to neutrinoless double beta decay, Phys. Rev. C 97, 065503 (2018).

[38] O. Azzolini et al., Search for neutrino-less double beta decay of ${ }^{64} \mathrm{Zn}$ and ${ }^{70} \mathrm{Zn}$ with CUPID-0, arXiv:2003.10840.

[39] N. Aghanim et al. (Planck Collaboration), Planck 2018 results. VI. Cosmological parameters, arXiv:1807.06209. 